\title{
PHOTOSENSITIVE POLYIMIDE APPLICATION FOR MULTI-CHIP PACKAGES
}

\author{
Tatsuo Inoue, Ken Seino*, Koetsu Tamura*, \\ Shin-ichi Hasegawa and Kohji Kimbara \\ Packaging Engineering Department, Computer Engineering Division \\ *Production Engineering Department , 1st Computer Division \\ NEC Corporation \\ 1-10, Nisshincho , Fuchu city , Tokyo 183, JAPAN
}

This paper introduces the photosensitive polyimide application for the NEC SX-3 Supercomputer. In case of supercomputers, sophisticated packaging technologies are essential to achieve fastest operations as well as to use highest-speed, highly integrated LSIs.

Wiring substrate which mounts and interconnects LSIs is the key to back up LSI's higher logical-operations.

The high speed interconnection wirings and high density LSI mounting are requested for the substrate.

The Multi-Chip Package using Photosensitive Polyimide had been developed to meet these demands.

$25 \mu \mathrm{m}$ wide, $75 \mu \mathrm{m}$ center-to-center spacings, four signal layers, $6 \mathrm{~ns} / \mathrm{cm}$ signal transmissions on a $225 \mathrm{~mm}$ square substrate have been achieved by using this photosensitive polyimide multi layer substrate technology.

\section{INTRODUCTION}

The large-scale scientific and technical calculations require computers that can execute operations at an ultra high speed. The NEC SX-3 system is a supercomputer developed for such scientific and technical calculations [1]. It's maximum performance is 5.5 Giga-Flops of 
uniprocessor. The key factors in reducing machine cycle time are the use of high speed VLSIs and reduction of signal propagation delay which is led by the new circuit packaging technology.

A VLSI chip is packaged in a chip carrier called the FTC(Flipped TAB Carrier). A high-density Multi-Chip Package(MCP) consists of a Multi-Layer Substrate(MLS) with a maximum of 100 FTCs and $11,540 \mathrm{I} / O$ pins. The MLS is $225 \mathrm{~mm}$ X $225 \mathrm{~mm}, 5.5 \mathrm{~mm}$ thick ceramic substrate with a fine line multilayer wiring part consists of 7 polyimide insulating layers and 8 conductor layers. A multilayer board mounts the above MCPs with new zero insertion force connectors. A sophisticated and reliable water cooling technique is used to cool the package efficiently. Water circulates from a cooling unit to liquid cooling modules that cover the MCPs. This system has a cooling capacity of up to $4 \mathrm{KW}$ heat dissipation of the MCP.

\section{MULTILAYER SUBSTRATE}

The multilayer substrate for supercomputers must have a high-speed and a high-density signal wiring . The signal propagation delay $t$ is given by the relationship

$$
t=\sqrt{\varepsilon r} / c
$$

where $\varepsilon r$ is a dielectric constant of the insulating material and $c$ is the speed of light. This formula shows the necessity for the use of lower dielectric constant material for insulating layers. The polyimide resin has a low dielectric constant of about 3.5. Moreover, the polyimide resin has very smooth surface and fine via hole formability [2].

The SX-3 Supercomputer is an evolutionary extension of the SX-2 which was the first supercomputer to use the polyimide-ceramic multilayer substrate [3]. Figure 1 illustrates cross-section of the Multi-Layer Substrate (MLS) used for the SX-3. The thinly laminated multilayer for

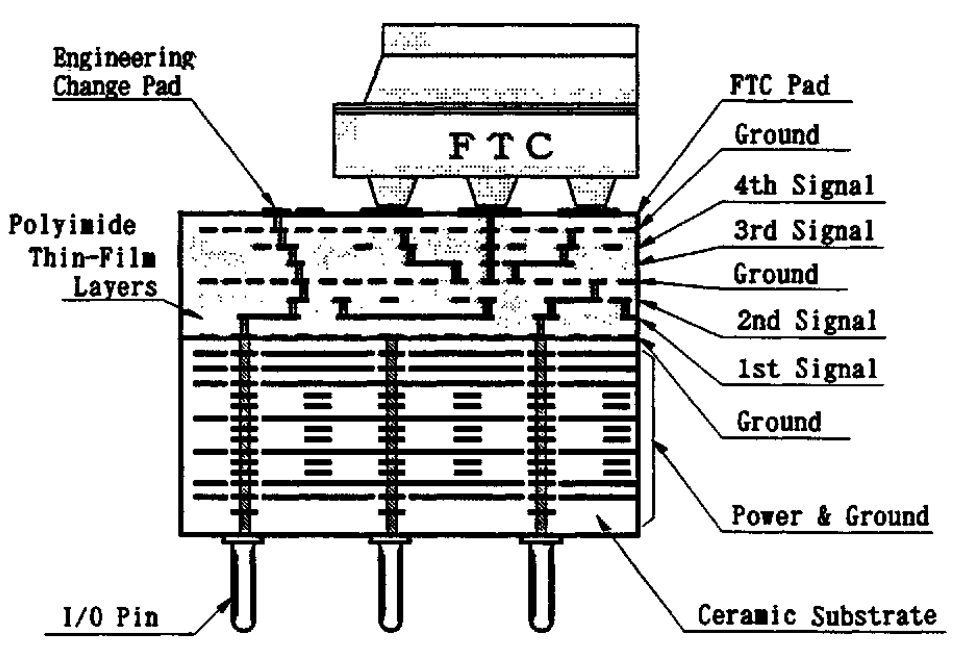

Fig.1. MLS Cross Section

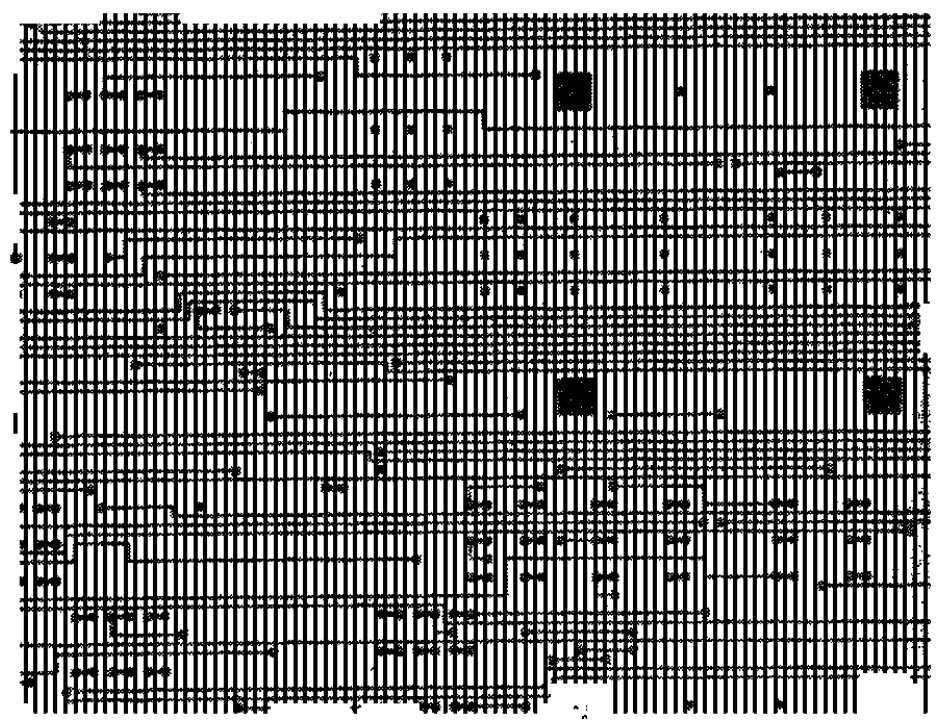

Fig. 2. Fine Signal Lines 
wiring with polyimide insulating layers has 8 conductive layers with 4 signal layers. The signal wiring layer pitch is $75 \mu \mathrm{m}$, wiring width $25 \mu \mathrm{m}$, and via hole in the polyimide layer $60 \mu \mathrm{m}$ square. Figure 2 shows the fine lines on the substrate. The low dielectric constant of polyimide resin and a fine line of the signal lines allowed high speed transmission of signals and high density of wiring connection. The size of MLS is $225 \mathrm{~mm} \mathrm{x} 225 \mathrm{~mm}$ and the ceramic substrate thickness $5.5 \mathrm{~mm}$. Figure 3 shows the overview of the

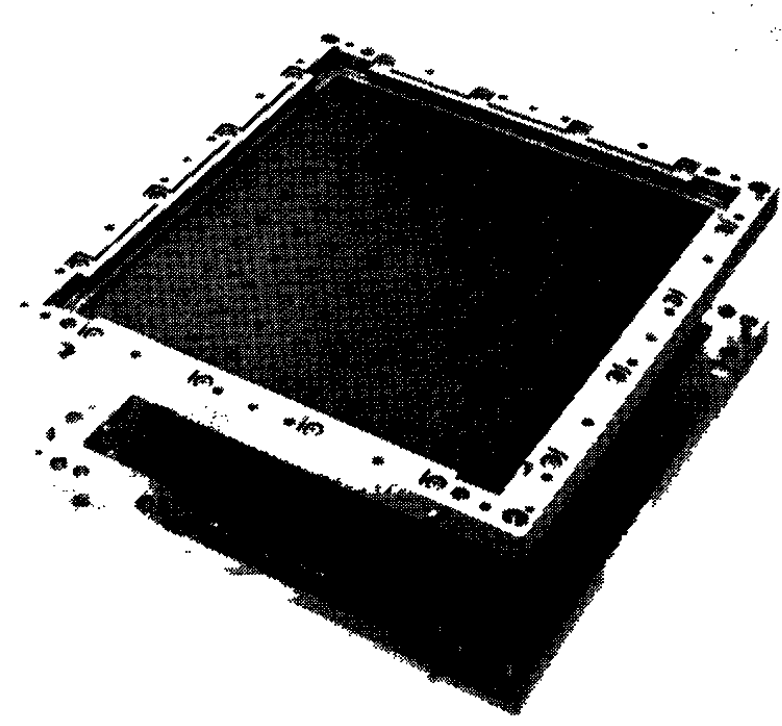

Fig. 3. MLS Overview MLS.

\section{MULTILAYER SUBSTRATE MANUFACTURING TECHNOLOGIES}

We developed the photosensitive polyimide technology for fabrication of a polyimide multilayer substrate with signal wiring width of $25 \mu \mathrm{m}$, wiring pitch of $75 \mu \mathrm{m}$, and via hole size of $60 \mu \mathrm{m}$. We used Toray's photosensitive polyimide "Photoneece ${ }^{\circledR}$ " to create fine via holes in the polyimide insulating layer. The photosensitive polyimide facilitates a patterning process compared with the non-photosensitive polyimide. The size of via holes in the polyimide insulating layer used in the SX-3 is $60 \mu \mathrm{m}$ square. The thickness of an insulation layer between the wiring layers is $20 \mu \mathrm{m}$. The projection type exposure is used for exposure. We did not use a contact type exposure machine since we could not make sufficient mask contact due to the use of ceramic substrate. The development is used for ultrasonic dipping.

\section{PROPERTIES OF POLYIMIDE}

For development of polyimide multilayer substrates, we evaluated the following polyimide resin characteristics:

[1] Polyimide film strength

With the polyimide multilayer substrate, polyimide fine wiring layers are piled up one by one on the ceramic substrate. Therefore, the polyimide at the bottom layer is subjected most frequently to thermal stress due to repeated curing. Figure 4 shows the change in elongation, tensile strength, and modulus of the polyimide film by high-temperature storage $\left(400^{\circ} \mathrm{C}, 24 \mathrm{~h}\right)$. Photoneece ${ }^{\circledR}$ gives a large elongation with average $18 \%$ after 24 hours storage at $400^{\circ} \mathrm{C}$. No big 
change is found in the tensile strength and modulus. The structural analysis of Photoneece ${ }^{\circledR}$ film reveals that curing repeated 50 times corresponds to 7 hours high-temperature storage at $400^{\circ} \mathrm{C}$. Consequently, it can be estimated that the elongation of $10 \%$ or more is obtained for Photoneece ${ }^{\circledR}$ after curing repeated 140 times.

As described above, the elongation of polyimide resin drops as it sustains thermal stress. When the elongation falls below $10 \%$, cracks tend to be break out in the polyimide layer during a process of forming a multilayer substrate.

These findings oblige us to decide the type of polyimide to be used for polyimide multilayer substrates by considering the drop in the elongation of the polyimide resin film by repeated curing.

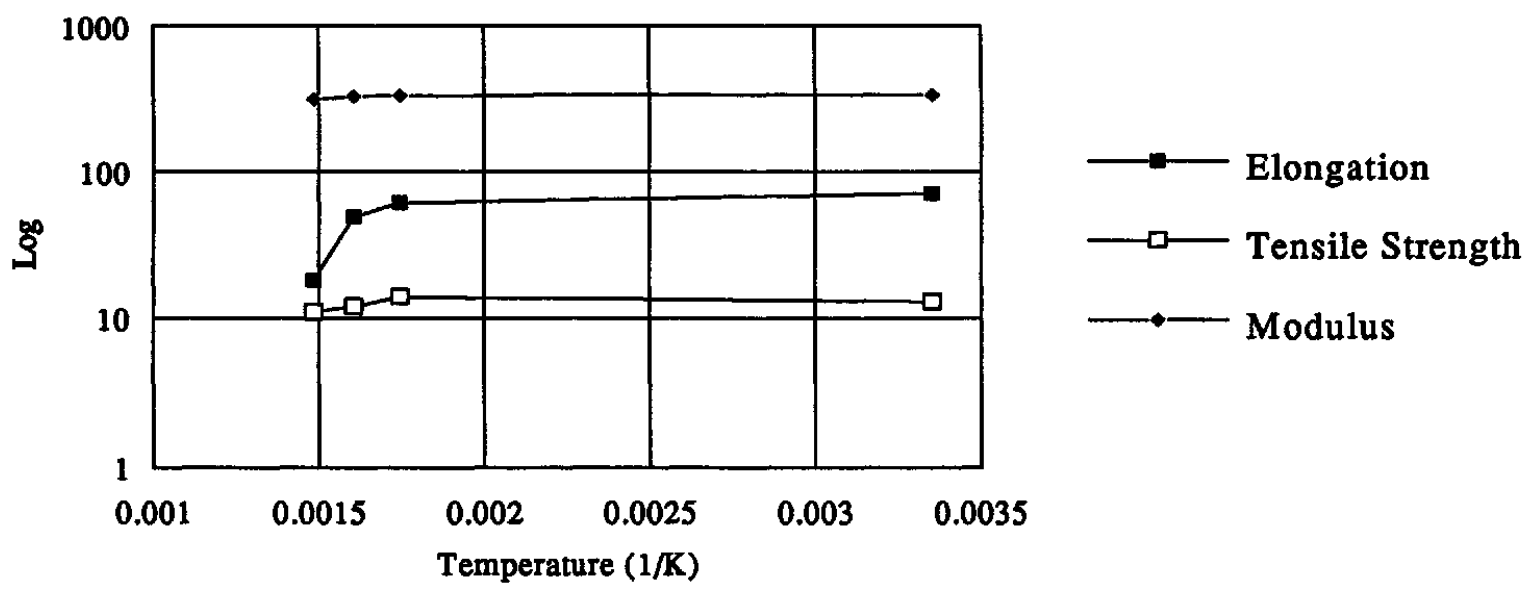

Fig.4 Elongation, Tensile Strength, and Modulus vs. Temperature

[2] Structural change in the polyimide film by repeated curing

The elongation of the polyimide film changes by repeated curing. We study this change from the polyimide film structure.

(1)Change in the glass transition point

Figure 5 shows the glass transition point by the DSC method. The glass transition point rises by $40^{\circ} \mathrm{C}$ by repeated curing 50 times. The displacement at the glass transition point also is decreased. This indicates the more curing is repeated, the less the molecular movement of polymers.

(2)Analysis by FT-IR ATR method

Figure 6 shows the difference spectrum in the FT-IR spectra between the polyimide film cured once and that cured 50 times repetitively. The differences between the spectra are marked near wave numbers $1240 \mathrm{~cm}^{-1}, 1500 \mathrm{~cm}^{-1}, 1720 \mathrm{~cm}^{-1}$ on the negative side and near $1620 \mathrm{~cm}^{-1}$ on the positive side. The decrease near $1240 \mathrm{~cm}^{-1}$ is due to the stretching vibration $\nu(\mathrm{C}-\mathrm{O}-\mathrm{C})$ of the aromatic ether. This is probably caused by a slight 
decomposition of the diphenyl ether bond due to heating. The decrease near $1500 \mathrm{~cm}^{-1}$ is by the absorption of the benzene ring and near $1720 \mathrm{~cm}^{-1}$ by that of the imide-carbonyl group, with the peaks changed probably by the decomposition of the diphenyl ether bond. The increase near $1620 \mathrm{~cm}^{-1}$ is weak and it is not certain whether it is attributable to the structural change. The N-H stretching vibration $\nu(\mathrm{N}-\mathrm{H})$ near $3270 \mathrm{~cm}^{-1}$ to be found when the N-R bond of the imide ring is decomposed is not observed in the current measurement. It is assumed no change occurred in the imide ring.

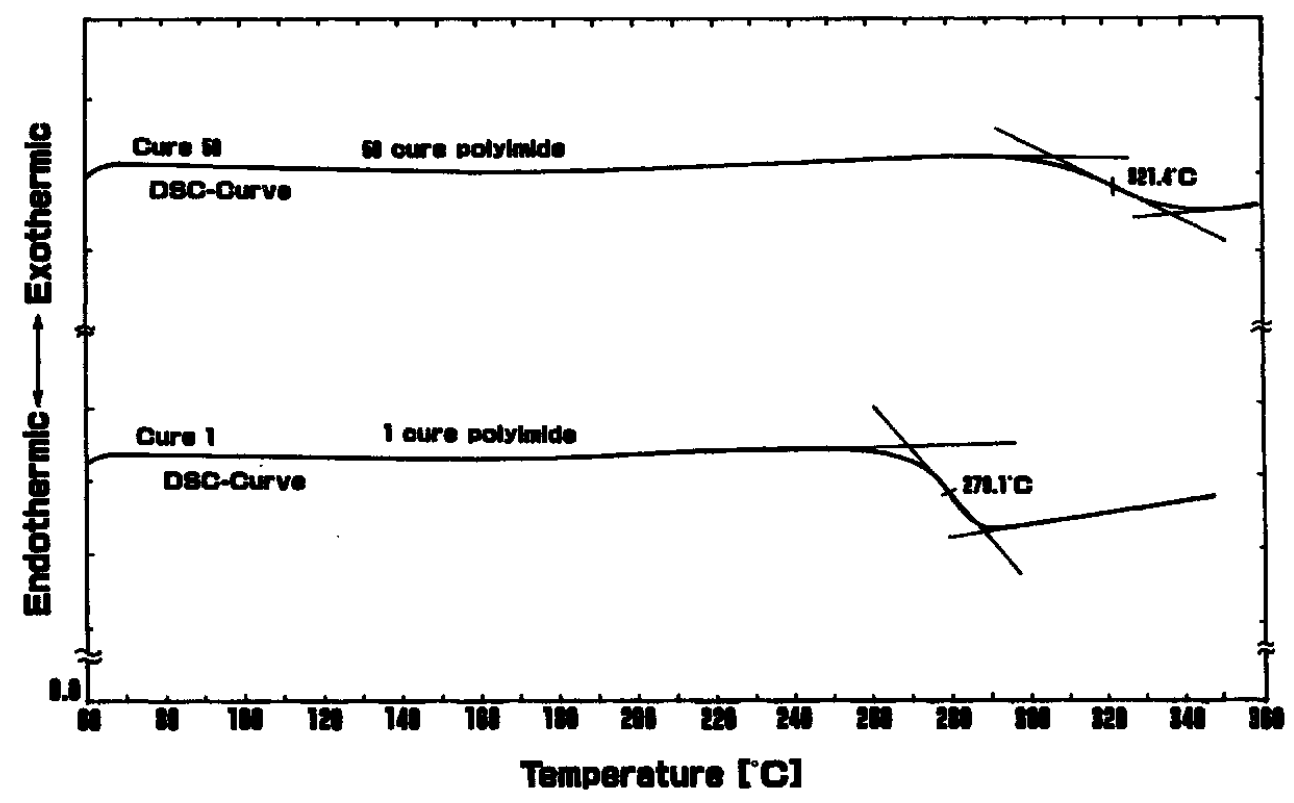

Fig. 5. The Difference of DSC Curves between 1 Cure \& 50 Cure Polyimide

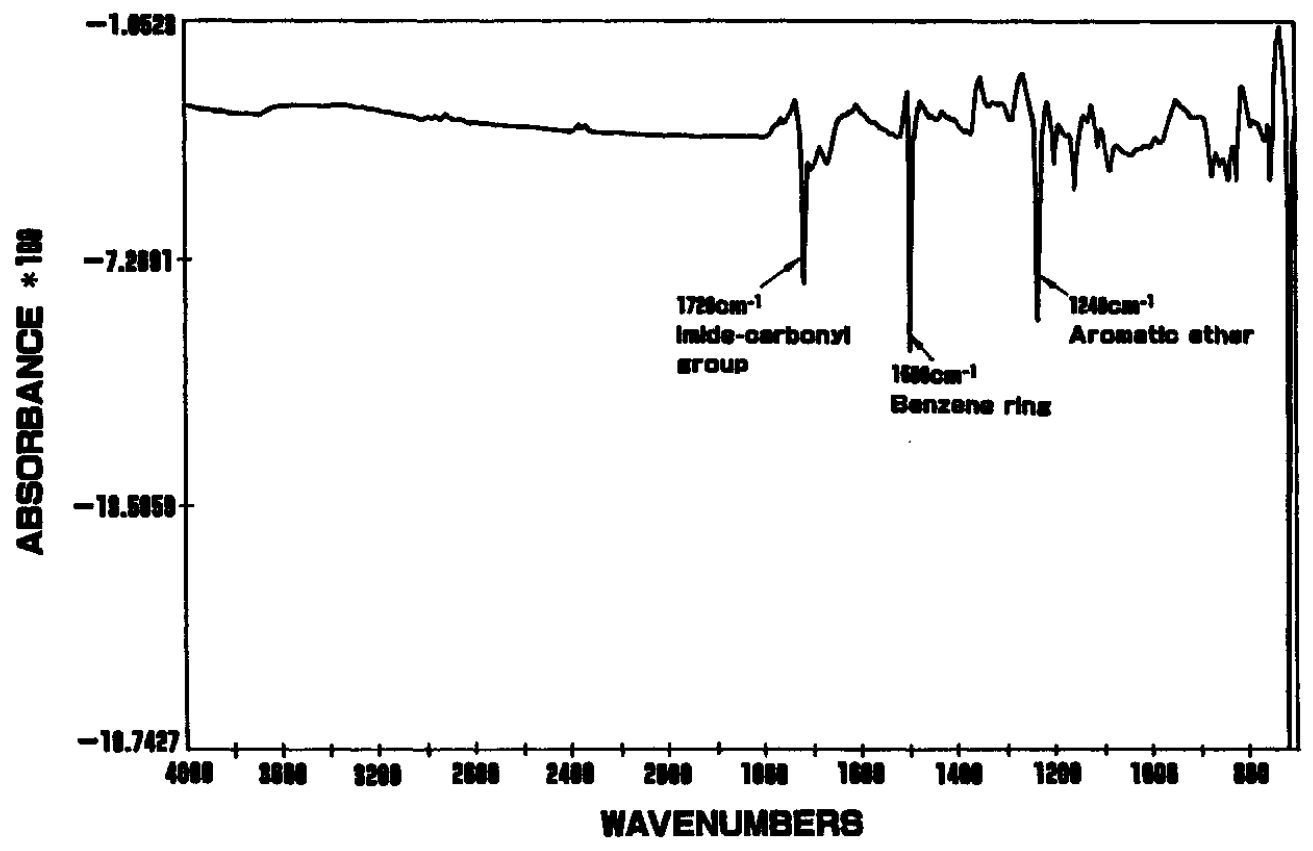

Fig. 6. The FT-IR Difference Spectrum between 1 Cure \& 50 Cure Polyimide 


\section{(3)Analysis by CP/MAS ${ }^{13} \mathrm{C}-\mathrm{NMR}$}

Figure 7 shows the difference spectrum between the polyimide film cured once and that cured 50 times repetitively. Signals come out strong at $125 \mathrm{ppm}$ and $132 \mathrm{ppm}$. This indicates the curing repeated 50 times has restricted the motion of the benzene ring. It appears that the stacking of polyimide benzene rings have progressed to build an aggregated structure.

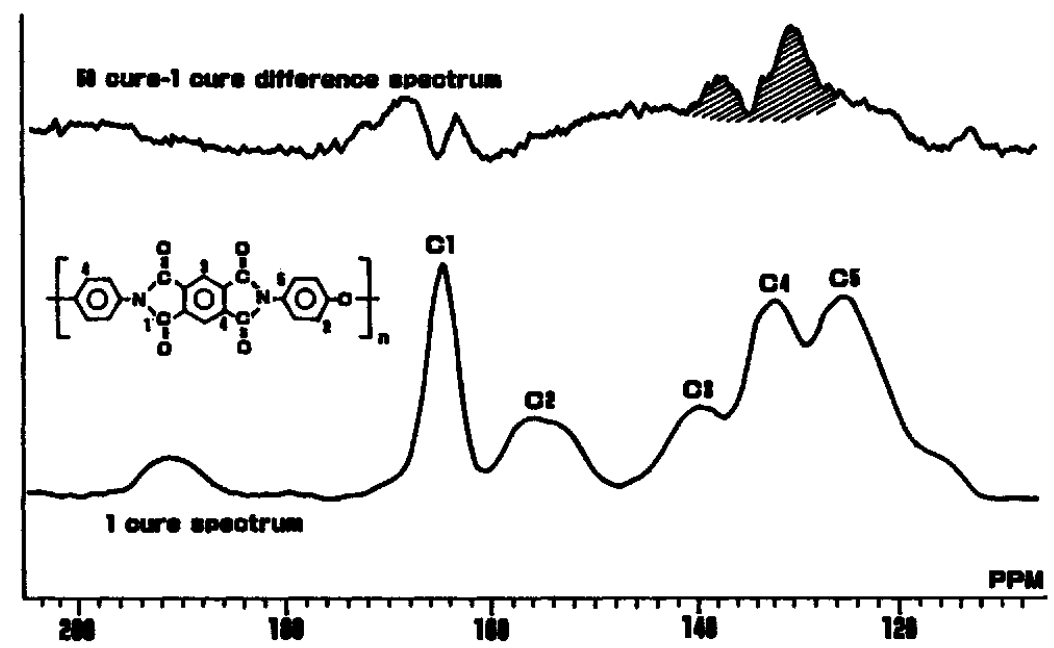

Fig. 7. The CP/MAS ${ }^{13} \mathrm{C}-\mathrm{NMR}$ Difference Spectrum between 1 Cure \& 50 Cure Polyimide

From these results of analysis, we believe the structural changes in the polyimide film by repeated curing to be as follows:

1. No big change in the polyimide molecule decomposition or bond.

2. Benzene ring stacking advances. As a result, the glass transition point rises and the film elongation comes to drop.

[3] Patternability of Photosensitive Polyimide

A ratio of $80 \%$ or more of the film retention after development is considered necessary for manufacturing use of photosensitive polyimide. Figure 8 shows the relationship between the dose of exposure and the film retention of photosensitive polyimide Photoneece ${ }^{\circledR}$. As shown in Figure 8 , the practical development conditions for photosensitive polyimide on the $225 \mathrm{~mm}$ square ceramic substrate are as follows:

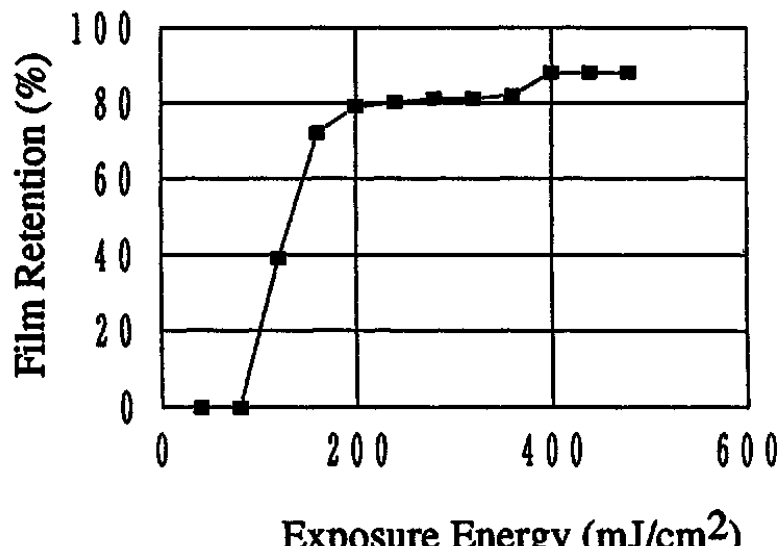

Fig.8 Photoresponse 
Prebake temperature : $74^{\circ} \mathrm{C}$

Exposure energy : $400 \mathrm{~mJ} / \mathrm{cm}^{2}$

Development time : $200 \mathrm{sec}$

Photoneece ${ }^{\circledR}$ is submitted to ultrasonic dip development. Therefore it is important to control the developer temperature and ultrasonic output. Figure 9 shows the SEM photograph of via hole.

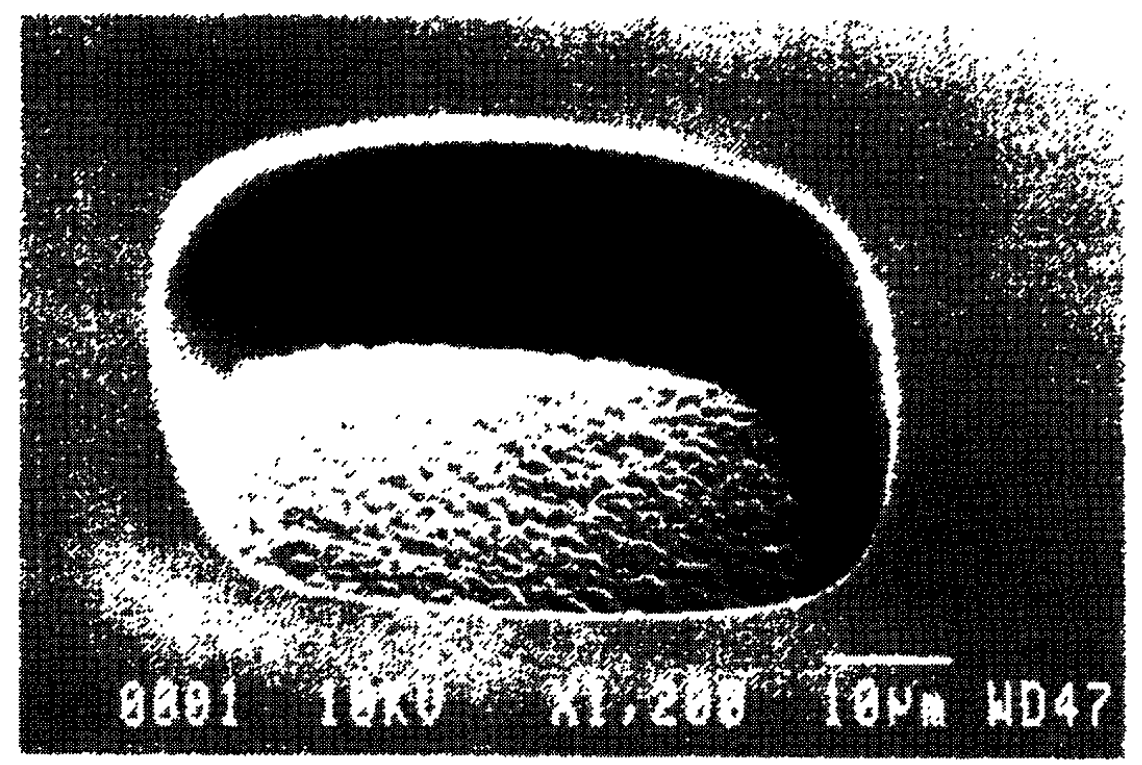

Fig. 9 SEM Photograph of Via Hole

\section{SUMMARY}

The NEC supercomputer SX-3 has been accomplished by the development of the following new technologies:

(1) High-speed , high-density LSI

(2) High-density, high-speed Multi-Chip Packages

(3) High-efficiency liquid cooling technologies

Particularly in the development of the high-density, high-speed Multi-Chip Packages, we established a process technology of forming on a large multilayer ceramic substrate fine wiring layers using polyimide resin with low dielectric constant. This technology was developed by advanced integration of the following technologies:

(1)Forming a fine pattern with photosensitive polyimide

(2)Forming a fine wiring pattern

Using these technologies, we completed large polyimide multilayer substrates for supercomputer with the following features:

(1) $25 \mu \mathrm{m}$ wide, $75 \mu \mathrm{m}$ center-to-center spacing high-density wiring

(2)Polyimide insulating layers $20 \mu \mathrm{m}$ in thickness and with $60 \mu \mathrm{m}$ square via holes 
(3)6 $\mathrm{nsec} / \mathrm{m}$ high-speed signal transfer

(4)11,540 input/output pins

(5)100 LSIs mountable

\section{ACKNOWLEDGMENT}

The authors are indebted to many people who have contributed to this project, especially to these members of the Packaging Engineering Department in Computer Engineering Division and the Production Engineering Department in the 1st Computer Division. We are also grateful to Toshihiko Watari Assistant General Manager of Computer Engineering Division, and Minoru Okano Manager of Packaging Engineering Department in Computer Engineering Division for much advice and guidance.

\section{REFERENCES}

1. A.Dohya, T.Watari and H.Nishimori, IEEE ECTC Conf., May 1990, p525

2. T.Watari and H.Murano, Proceeding of 35th ECC, May 1985, p192

3. K.Kimbara, A.Dohya and T.Watari, Mat.Res.Soc.Symp., Proc.Vol.167 , 1989 ,p33 